\title{
Evolution of the magnetic field in magnetars
}

\author{
J. Braithwaite and H. C. Spruit
}

Max-Planck-Institut für Astrophysik, Karl-Schwarzschild-Straße 1, Postfach 1317, 85741 Garching, Germany

e-mail: [jon;henk] @mpa-garching.mpg.de

Received 9 September 2004 / Accepted 12 December 2005

\section{ABSTRACT}

We use numerical MHD to look at the stability of a possible poloidal field in neutron stars (Flowers \& Ruderman 1977, ApJ, 215, 302), and follow its unstable evolution, which leads to the complete decay of the field. We then model a neutron star after the formation of a solid crust of high conductivity. As the initial magnetic field we use the stable "twisted torus" field which was the result of our earlier work (Braithwaite \& Nordlund 2006, A\&A, 450, 1077), since this field is likely to exist in the interior of the star at the time of crust formation. We follow the evolution of the field under the influence of diffusion, and find that large stresses build up in the crust, which will presumably lead to cracking. We put this forward as a model for outbursts in soft gamma repeaters.

Key words. instabilities - magnetohydrodynamics (MHD) - stars: magnetic fields - stars: neutron

\section{Introduction}

The soft gamma repeaters (SGRs) are characterised by continuous emission of X-rays of luminosity $10^{35}-10^{36} \mathrm{erg} \mathrm{s}^{-1}$ (Rothschild et al. 1994; Hurley et al. 1999) and X-ray outbursts, each lasting less than a second, but in total accounting for a similar luminosity to the continuous emission. These outbursts are extremely bright (up to $10^{42} \mathrm{erg} \mathrm{s}^{-1}$ ) and therefore super-Eddington $\left(10^{4} L_{\mathrm{Edd}}\right)$, and in three of these objects (the whole class contains only four known specimens) a much brighter outburst has been observed. In SGR 0526-66 an outburst on 5th March 1979 released X-rays to the tune of an estimated $4 \times 10^{44} \mathrm{erg}$, SGR $1900+14$ released $1 \times 10^{44} \mathrm{erg}$ in its 27th August 1998 outburst (Cline 1982; Mazets et al. 1999), while the most powerful event of all, on 27th December 2004, SGR 1806-20 produced an estimated $2 \times 10^{46} \mathrm{erg}$ (Palmer et al. 2005). These giant outbursts consisted of a short intense burst of a fraction of a second, followed by an afterglow lasting several minutes. Regular variability in the X-ray flux from these objects has been detected, which can most easily be interpreted as the rotational period, $P$. The periods range from 5 to $8 \mathrm{~s}$. If one measures the period over a long period of time, one can measure the rate of change of period $\dot{P}$ and hence calculate a characteristic age $P / \dot{P}$. For two SGRs, values of around 3000 years have been measured (Kouveliotou et al. 1998; Woods et al. 2000).

About 8 further objects have been classified as anomalous $X$-ray pulsars (AXPs) - their observational characteristics are essentially those of SGRs without outbursts. They emit X-rays at the same luminosity, rotate at the same speed (6 to $12 \mathrm{~s}$ ) and have the same characteristic ages. This similarity suggests that AXPs are nothing but SGRs in some kind of dormant state (Thompson \& Duncan 1996; Mereghetti 2000).

That most of these objects have been associated with young supernova remnants leads naturally to the hypothesis that they are neutron stars. The question of the nature of the energy source for their X-ray emission has been answered in several ways, although most authors now agree on a strong decaying magnetic field, the magnetar model, first proposed by Duncan \& Thompson (1992). According to this model, the observed $\mathrm{X}$-ray luminosity (in quiescence and in outburst) is powered by the release of magnetic energy in a very strong $\left(B>10^{14} \mathrm{G}\right)$ field. In contrast, the accretion and fossil-ring models have run into significant difficulties in recent years.

The evidence for such a strong field is the rapid rotational spindown of these objects (see, for example, Shapiro \& Teukolsky 1983 for an explanation of the relevant physics). A magnetic field of the inferred strength will contain about $10^{47}$ erg - enough to account for the observed luminosity for around $10^{4}$ years. This is significantly more energy than these stars contain in the form of rotational kinetic energy - a neutron star rotating with a period of $6 \mathrm{~s}$ will contain just $5 \times 10^{44} \mathrm{erg}$. The spindown luminosity (the rate of change of this rotational kinetic energy) of SGRs is measured at $10^{34} \mathrm{erg} \mathrm{s}^{-1}$, between one and two orders of magnitude too small to account for the X-ray luminosity. These objects can therefore, unlike classical pulsars, not be powered by spindown alone. This is the main motivation for the magnetic decay model for SGR/AXP emission.

In this paper, we develop the magnetic decay model a little further by presenting stable field configurations obtained from 3D numerical simulations, and by computing the pattern 
of crustal stress that develops when such a field evolves by diffusion.

\subsection{Powering by magnetic field decay}

In the magnetic decay model the primary cause of the decay is energy release by some form of rearrangement of the magnetic field configuration in the star. Most of the magnetic energy is contained in the interior, and a smaller (but possibly comparable) amount in the atmosphere. Rearrangements therefore release energy both in the atmosphere and in the interior. To the extent that this is a slow continuous process ("creep"), it powers quiescent emission, both in thermal form (heating of the interior) and non-thermal (the energy released in the atmosphere). Apparently, some rearrangement takes place suddenly ("fracture"). The atmospheric energy release in such an event powers the observed outbursts while the internally dissipated energy leaks out more slowly, adding to the quiescent emission (Thompson \& Duncan 1996). A straightforward extension of this interpretation is the possibility that the outburst episodes actually involve cracking of the neutron star crust and consequent release of magnetic energy in the atmosphere (Thompson \& Duncan 1995). A slow steady change in the interior field, with the surface field kept in place by the solid crust, would slowly build up magnetic stress at the crust/core boundary, which is released in crustal quakes.

\subsection{Origin of the field}

The magnetic fields of neutron stars were probably already present at birth (the possibility of a later origin by thermomagnetic effects seems less likely, cf. Blandford et al. 1983). One possibility could be convection in the proto-neutron star, when unstable density gradients are produced by neutrino cooling. Possible field strengths up to $10^{15} \mathrm{G}$ may be generated by a convective dynamo (e.g. Thompson \& Duncan 1993). Another possibility is that the magnetic field is formed by compression during core collapse, from a field already existing in the progenitor star.

In either case, the magnetic field formed is likely to be out of equilibrium and unstable directly after the collapse, raising the question how much of the magnetic field can survive in the subsequent evolution when the star is still fluid. Once a solid crust has formed we may assume that at least the surface field is frozen, with further evolution taking place only on a much longer diffusive time-scale. It is thought that the crust will not begin to form until around $100 \mathrm{~s}$ after the collapse. If the field in the interior is of order $10^{15}$ Gauss, the Alfvén crossing time will be of order $t_{\mathrm{A}}=\sqrt{4 \pi \rho} R_{*} / B \approx 0.1 \mathrm{~s}$. Thus any unstable field has had ample time to either evolve, either into a stable configuration of lower energy (if this exists) or decay to nothing, before it can be "frozen in" by the crust.

This estimate is modified a little when we take into account that the star is likely to be rapidly rotating, with a period $P$ which may be shorter than the Alfvén crossing time $t_{\mathrm{A}}$. In this case, the magnetic instability time-scale is lengthened by a factor $t_{\mathrm{A}} / P$. For a plausible rotation period of $10 \mathrm{~ms}$, the instability

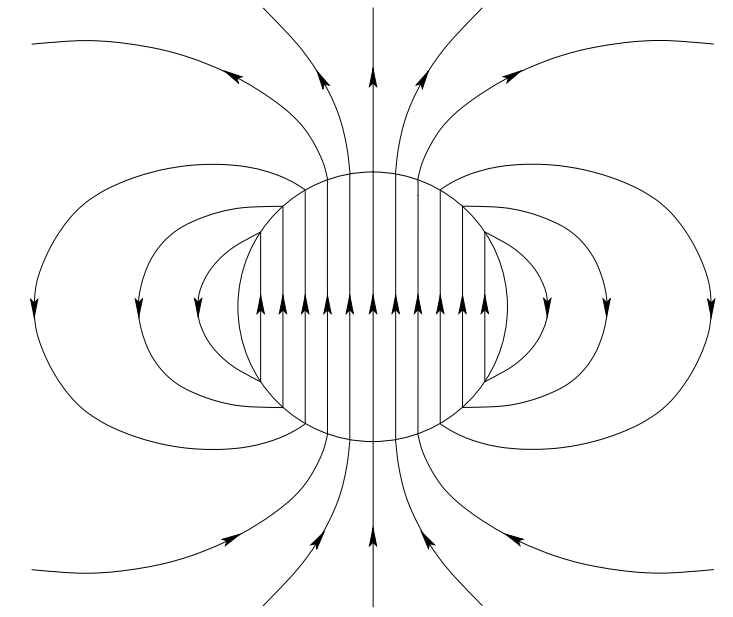

Fig. 1. The Flowers-Ruderman field.

time-scale is then increased to $\approx 1 \mathrm{~s}$, still short compared with the time till crust formation.

An important question is thus what happens to an initially unstable field configuration, and if/how it can evolve into a stable form.

\section{Stable and unstable field configurations}

We are interested in finding a field configuration which could account for the behaviour of these neutron stars. It is unclear what kind of field a neutron star may be born with, or rather, what kind of field it will have when the crust has formed. In a differentially rotating star, purely toroidal field configurations (azimuthal fields) form naturally from a weak initial field by winding up of field lines (Spruit 2002, and references therein). The proto-neutron star may well be a strongly differentially rotating object that would nicely wind up field lines. It is known, however, that predominantly toroidal fields are all unstable on an Alfvén crossing time (Tayler 1973; Acheson 1978; Pitts \& Tayler 1986; Braithwaite 2005).

At the other extreme, one can think of a purely poloidal field (field lines confined to meridional planes, $B_{\phi}=0$ ). Such a field is again known to be unstable on an Alfvén crossing time (Wright 1973; Markey \& Tayler 1973, 1974) if it has field lines that are closed inside the star. If the field lines close only outside the star, the stability analysis is slightly different but the result the same. A well-known simple example of this case is a configuration consisting of a uniform field inside the star, with a matching dipole outside the star (Fig. 1). An argument by Flowers and Ruderman shows why this field is unstable in the absence of a crust. Since this example has played some role in the discussion of neutron star magnetic fields, we study it in some detail below, with a numerical simulation in Sect. 3 .

\subsection{An unstable field configuration}

Flowers \& Ruderman (1977) consider a uniform field in the neutron star interior with a potential field outside it. They find this to be unstable on a dynamic time-scale (Alfvén crossing time-scale). A simple way to understand this (see Fig. 2) is to 

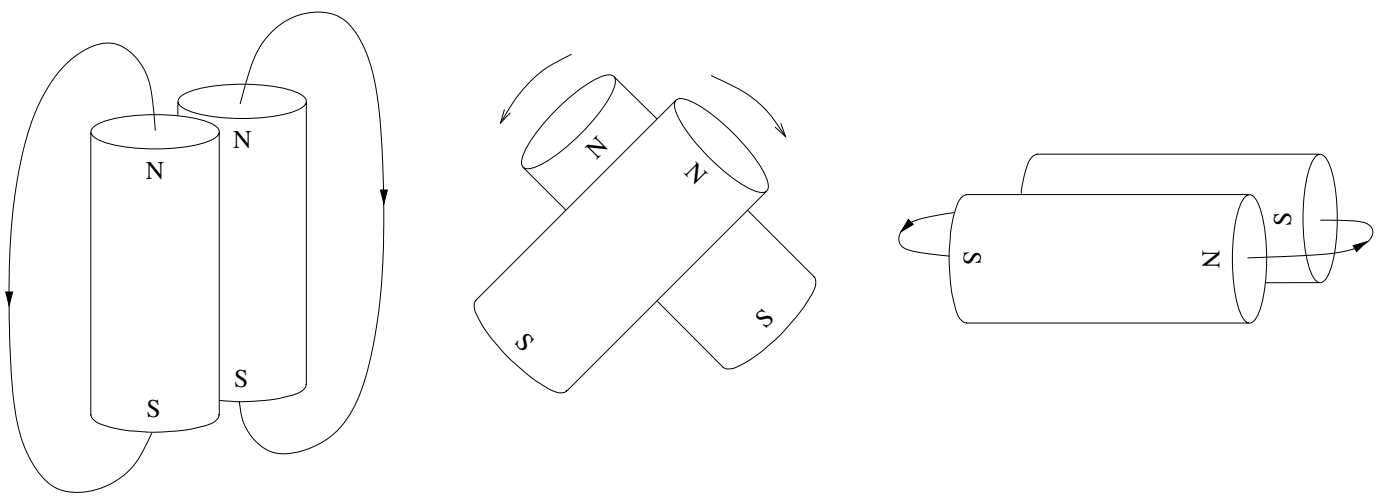

Fig. 2. Flowers-Ruderman argument for the instability of a star with a uniform interior magnetic field. Two bar magnets are free to rotate about a common axis. If pointing in the same direction, they are unstable and will rotate until pointing in opposite directions.

imagine the uniform field as a collection of parallel bar magnets. Two bar magnets side by side, pointing in the same direction will repel each other, since the north pole of one will be next to the north pole of the other. They will tend to rotate so that the north pole of one is next to the south pole of the other, reducing the magnetic energy outside but not inside the magnets. In a fluid star, without stabilising solid-state forces, the equivalent process would be to cut the star in half along a plane along the field lines, and rotate one of the halves by $180^{\circ}$. This reduces the energy in the potential field outside the star in the same way as in the case of the bar magnets, whilst all energy forms inside the star (magnetic, thermal and gravitational) remain unchanged, also as in the case of the bar magnets.

Flowers and Ruderman then consider ways in which a field may be stabilised. Firstly, the solid crust of the neutron star, if it forms quickly enough, could prevent the decay of a field. Secondly, the addition of a toroidal component could stabilise the field, because its energy would necessarily increase during the motion depicted in Fig. 2. To understand this, imagine wrapping an elastic band around the two bar magnets. Rotating the magnets now will stretch the elastic band, requiring energy. Field lines behave in a similar way, because to stretch them also requires energy.

\subsection{Stable field configurations and the role of helicity}

It was predicted by Prendergast (1956) that a stable field inside a star may consist of a polar dipole component stabilised by a toroidal component of comparable strength. This principle is applied with success in the design of fusion reactors. Recently it has become possible to follow the evolution of an arbitrary field with numerical magnetohydrodynamics. This was done by Braithwaite \& Nordlund (2006, hereafter Paper I) - for a summary of the results see Braithwaite \& Spruit (2004) - where the related problem of the origin and evolution of the fields of magnetic A stars was studied. The main result from this work is the emergence of a stable field configuration, independent of the initial field configuration.

The stable field is approximately axisymmetric, and does indeed have both poloidal and toroidal components of comparable strength. The form of the resulting field is always similar: a torus-shaped configuration embedded inside the star,

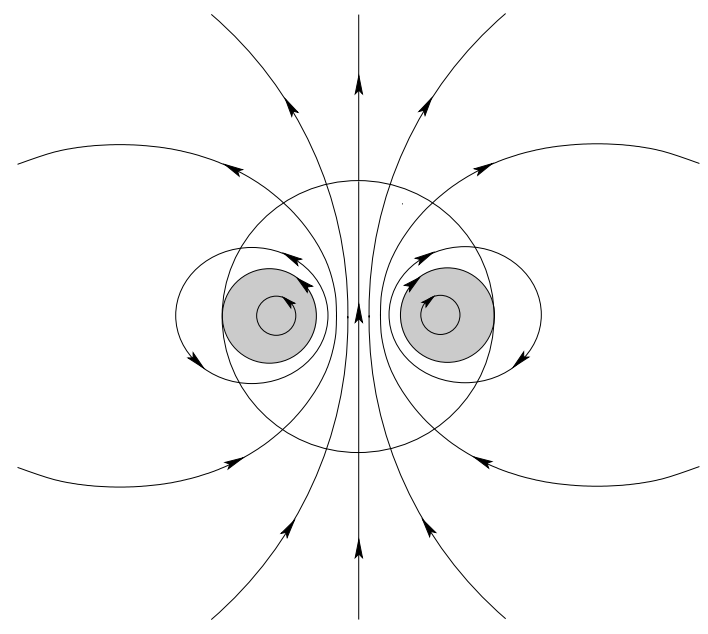

Fig. 3. The stable linked poloidal-toroidal torus field. Poloidal field lines are drawn; the shaded areas represent the azimuthal field component.

with an approximately dipolar field connected to it outside the star. Most of the field lines, projected onto a meridional plane, are closed inside the star, but with some extending outside it (see Fig. 3).

To the extent that the atmospheric field is close to a potential field, it does not support twists or internal torques. For an axisymmetric configuration, this means that the azimuthal field component vanishes everywhere along a field line that extends outside the star. The azimuthal field component is thus confined to a torus defined by the lines that are closed inside the star. The field lines extending outside the star define an approximate dipole, possibly somewhat offset from the centre. This is in fact just what is observed in the magnetic A stars. This apparent dipole is not representative of the configuration as a whole: in the interior, it looks very different. This solves one of the theoretical puzzles of the fields observed in A stars: they would be violently unstable if the nearly dipolar fields seen at the surface if were representative also of the interior.

The strength of the field found in the simulations depends on initial conditions and, as should be expected, the orientation of the final configuration is random (at least in the absence of rotation). 
The conclusion from these results is that an arbitrary unstable initial field does not in general decay completely, but gets stuck in a stable equilibrium at some amplitude. That this should be so becomes understandable (Moffat 1990) in terms of the approximate conservation of magnetic helicity. If $\boldsymbol{A}$ is a vector potential of the field $\boldsymbol{B}$, the quantity

$H=\int \boldsymbol{A} \cdot \boldsymbol{B} \mathrm{d} V$,

where the integral is taken over the volume inside a magnetic surface, is called the magnetic helicity (Woltjer 1958). (Issues relating to the gauge used for $\boldsymbol{A}$ are involved, but of no consequence in the present context.) The helicity is conserved under arbitrary displacements, as long as magnetic diffusion and reconnection may be neglected.

If an initial field configuration has a finite helicity, it can not decay further than the lowest energy state with the same helicity (again as long as reconnection may be ignored). In practice, reconnection cannot be ignored since diffusion will be important on small length scales, and rapid reconnection can happen through regions with dynamically-generated small length scales. Nevertheless, it is found that in practice helicity conservation is often a good approximation on a large scale, even when reconnection takes place on smaller scales inside the configuration.

A second cause for lack of conservation of helicity is the tenuous atmosphere of the star, which does not support significant amounts of twist in the field configuration. Magnetic helicity can therefore "escape" through the surface. An example where such a process can be observed is the field configuration in the solar corona. Most of the time there is little evidence of twisted or non-force-free fields, in spite of the continuous rearrangement of field lines at the surface caused by the magnetic activity cycle. Loss of helicity through the surface is observed in the form of dynamic events such as prominence eruptions, or in a quiescent form in large scale structures like polar crown prominences (Low 2001, and references therein).

We find that this process may also be important for the relaxation to the stable torus configuration in the present calculation and those reported in Paper I. In calculations where loss of magnetic field through the surface is prevented by perfectly conducting boundary conditions, the final field configurations were found to be noticeably different.

In spite of these sources of helicity loss, it appears that the tendency towards conservation is still strong enough for a stable equilibrium to develop, at least in many cases. In this interpretation, the orientation and strength of the final configuration of an unstable magnetic field in a fluid star is thus closely connected with the helicity that happens to be present in the initial configuration.

The stable field produced in this way then evolves on a longer time-scale, under the influence of diffusion, as a sequence of quasi-static, stable equilibria. In the simulations, this is observed as a slow outward expansion of the configuration. In the process, the field lines that are closed inside the star loose their azimuthal field component as soon as they cross the surface of the star. As the azimuthal field decreases in this way, and the poloidal component starts dominating, the configuration eventually becomes dynamically unstable and decays to a low value (see Paper I for further discussion).

\section{Instability of a dipolar field}

As described in Sect. 2.1, a dipolar field in a ball of conducting fluid is expected to be dynamically unstable, but the argument given does not say anything about how the magnetic field will evolve in the non-linear regime. We use a 3D numerical simulation to see how the field evolves under this instability, and what it evolves into. The code used is that of Nordlund (see Nordlund \& Galsgaard 1995, for a detailed description). The setup employed for the present problem is described in detail in Paper I. In short, we model a self-gravitating ball of perfectly conducting (up to numerical diffusion) plasma. The initial model has the density and temperature distribution of a polytrope of index $n=3$. The equation of state is an ideal gas with constant ratio of specific heats $\gamma=5 / 3$. The model is thus strongly stably stratified. This model is embedded in a low-density atmosphere with an assigned poor conductivity, such that the field in the atmosphere stays close to a current free configuration, which adjusts quickly to the changing magnetic field distribution at the surface of the star. We start with a field of uniform strength and direction within the star, and a potential field in the atmosphere. This field is illustrated in Fig. 1. A spatial resolution of 144 cubed is used here.

In principle, we could have modelled a neutron star more accurately by using a more realistic equation of state. However, this would have involved a fair amount of work and it was felt that the result would not be affected significantly by using the equation of state of an ideal gas since it leads to nearly the same constraints on fluid motions.

Since the evolution of the field advances on an Alfvén timescale, the evolution slows down as the field decays. This is impractical for numerical reasons, since the minimal time step is governed by the sound speed, which stays the same. The entire evolution of an unstable magnetic configuration, however, takes place on an Alfvén time scale. Field configurations which are the same except for an amplitude factor $\alpha$ will therefore evolve in the same way, with only the speed of evolution differing by a factor $\alpha$. Except at very high field strength, when the Alfvén speed becomes comparable to the sound speed, and on small length scales, where diffusion becomes important, the amplitude of the field thus comes in only through the time-scale on which the field evolves. We make use of this by rescaling the field strength periodically by an overall factor, so as to keep the total field energy approximately constant. By keeping track of the rescaling factors applied, the correct field strengths and time axis can be reconstructed.

Since, for numerical reasons, the magnetic energy density in the calculations is only a factor 30 or so smaller than the thermal energy density, and diffusion does have an effect on small scales, some error is introduced by this rescaling process. Test results given in Paper I show that the process is accurate enough for the present purpose, in which the final state reached by the field configuration is of more interest than the exact dynamical evolution to this state. 

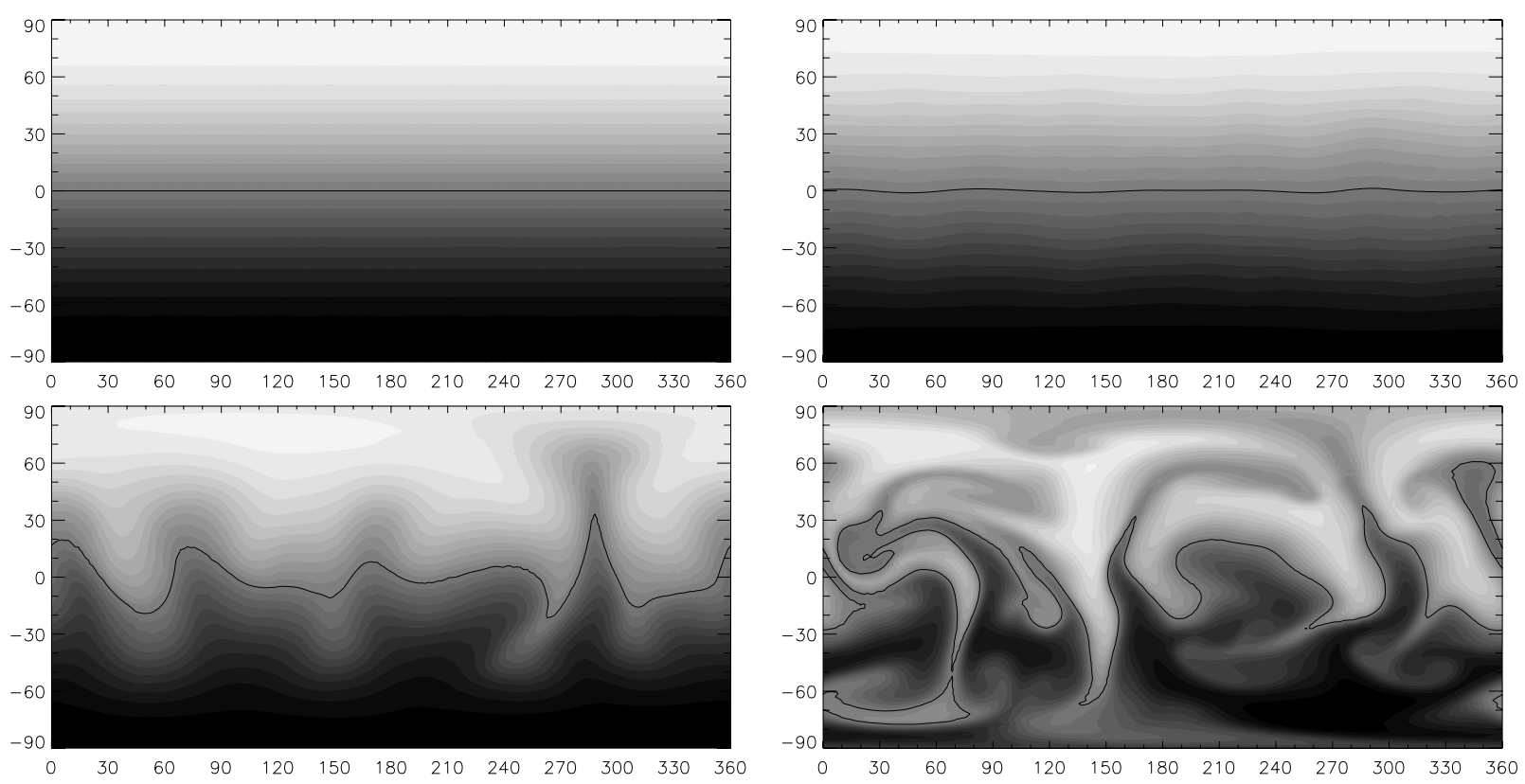

Fig. 4. Evolution of the magnetic field at the stellar surface. The initial state is a uniform magnetic field in the interior and a potential field in the atmosphere (cf. Fig. 1). The frames are taken at times: $t=0,4.4,5.0,5.7$ (top-left, top-right, bottom-left, bottom-right), the time unit used being the Alfvén crossing time. The radial component $B_{\mathrm{r}}$ is represented by light (positive) and dark (negative) shading. Also plotted, in black, is the line where $B_{\mathrm{r}}=0$.

\subsection{Result}

As expected, the field is unstable and decays over a timescale of a few Alfvén crossing times. One of the best ways to visualise the results is to look at the radial component of the field on the surface of the star. This is plotted in Fig. 4, projected in a simple manner onto two dimensions by mapping longitude to the $x$ coordinate and latitude to the $y$ coordinate. Field lines of the configuration are shown in Fig. 5.

The initial field was of such a strength that the Alfvén crossing time (given by $\sqrt{4 \pi \rho} R_{*} / B$, where $\rho$ and $R_{*}$ are the density and radius of the star) is roughly the same as the temporal separation of the last three frames in Figs. 4 and 5. This confirms that the field decays on an Alfvén time-scale.

The decay of the field continues for the entire duration of the calculation, without any sign of levelling off. This can be understood in terms of the magnetic helicity introduced in Sect. 2.2. The initial state used in this example has zero magnetic helicity, hence helicity conservation does not impose a lower limit on the final-state field. In principle, it is of course possible that stable field configurations exist with zero total helicity. An example could consist of two concentric tori with fields twisted in opposite directions. Apparently, such configurations are not easily reached in the present case.

Another caveat concerns the degree to which helicity is actually conserved. Since the atmosphere does not support twists in the field, helicity can be lost through the surface of the star. Even if there is no net helicity to being with, random fluctuations in twist propagating through the surface might leave the interior with a finite amount of helicity as a statistical accident of the complicated nonlinear evolution of the field. If such a process actually takes place, it has not been detectable at the sensitivity of the present calculations.

\section{Evolution of a stable twisted torus field in a neutron star with a solid crust}

In earlier study of stellar magnetic fields (Paper I; see also Braithwaite \& Spruit 2004) we found stable magnetic fields that can exist in the interior of a star. This field is a nearly axisymmetric, twisted torus shape. We found that such a field in an A star will slowly diffuse outwards until becoming unstable at some point. It is not known what kind of field may exist in a neutron star after it is formed, but it takes only a few Alfvén crossing times to form the torus configurations found in Paper I. During the first minutes after the formation of the neutron star in a core collapse the star is still hot and completely fluid. A magnetic field in it would relax in much the same way as in the A-star simulations reported in Paper I. For internal field strengths above $10^{12} \mathrm{G}$, corresponding to surface dipole fields above about $10^{11} \mathrm{G}$, the Alfvén crossing time would be short enough for the evolution to reach the stable torus equilibrium before the crust forms. After the solid crust has formed, the magnetic field in the fluid interior will continue to evolve under the influence of diffusion, and stress will build up in the crust owing to the Lorentz forces, possibly leading eventually to cracks, rather like the processes in the Earth's crust. This buildup of stress can be modelled with our numerical simulations.

The mechanical properties of the crust are not well understood and no attempt is made here to model its behaviour. We simply look at the Lorentz forces acting on it before it cracks, not at the cracking process itself.

The setup of the MHD code is described in Sect. 3 and in detail in Paper I. For this calculation we use the twisted torus as the initial field, precisely that field which is produced by the evolution with the same code of a random initial field. Since 

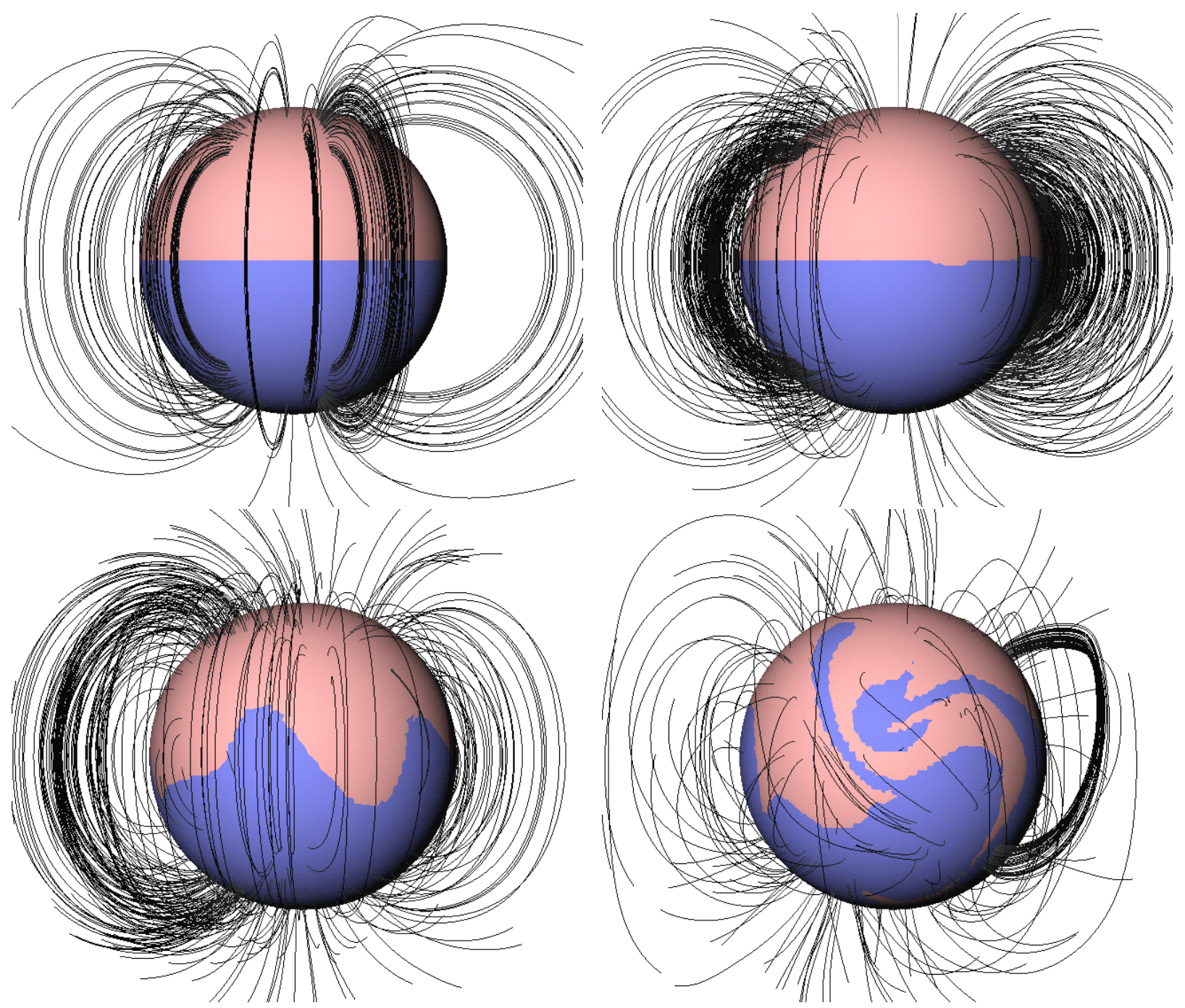

Fig. 5. Field lines in the stellar atmosphere. Shading on the stellar surface shows the sign of $B_{\mathrm{r}}$ (light positive, dark negative). The four panels correspond to the same times as those in Fig. 4.

we are only interested in what happens inside the star and the crust, and not in what happens in the atmosphere, we model a smaller volume than was modelled in our previous study the computational box is a cube of side $2.2 R_{*}$, as opposed to the $4.5 R_{*}$ used in the previous study and in the runs described in Sect. 3.1.

To the code which we have used previously we just need to add the solid crust. We model this as a zone between two radii in which the velocity field is held at zero (between $r=0.86 R_{*}$ and $r=1.07 R_{*}$ where $R_{*}$ is the radius of the star) and in which the magnetic field is held constant.

\subsection{Visualising and understanding the results}

We are wanting to look at the stresses that build up in the solid crust, as these will eventually cause the crust to crack. In a real star with an infinitely conducting crust and a diffusive fluid interior, we would expect to see a discontinuity in the field at the lower boundary of the crust, and a current sheet. In a more realistic crust of high but finite conductivity, the field lines would bend within a thin layer at the bottom of the crust, without any discontinuity in the magnetic field. The Lorentz force created by the change in the field will act on the crust in this thin layer. This is illustrated in Fig. 6.

At the upper boundary we would not expect to see any significant discontinuity since the field outside is essentially a potential field and will consequently remain constant after the crust has formed.

An MHD code of the type used here cannot model surface currents, yet we are trying to model a crust of infinite conductivity, in which the magnetic field is held constant. In order to look at the stress acting on the crust, therefore, we need to integrate the Lorentz force over a layer of at least a few grid spacings either side of the lower boundary of the crust. But what kind of Lorentz force are we interested in?

At the moment when the crust freezes, there will already be a non-zero Lorentz force. If we express this force as the divergence of a scalar plus the curl of a vector, the former will already be balanced by pressure (buoyancy) forces. The latter will give rise to fluid currents which will then have the effect of reducing the driving force itself - we can therefore expect this component of the force to be small at the moment when the 

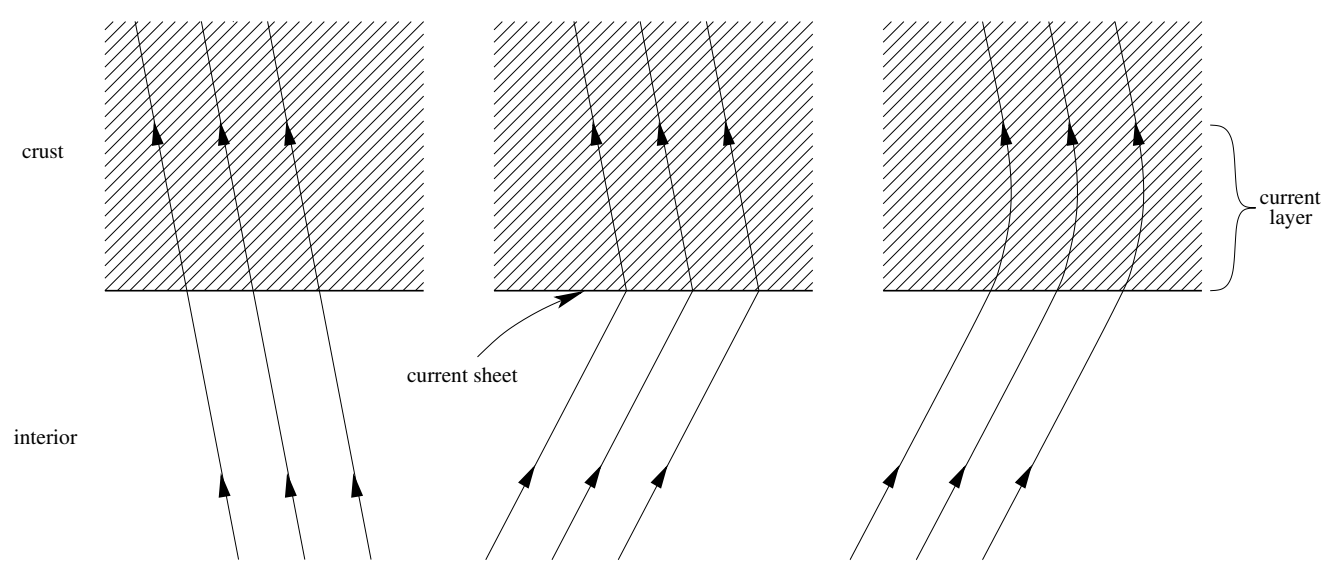

Fig. 6. Diagram showing the movement of field lines. On the left, the field lines at the moment that the crust freezes. In the middle, after the field in the interior of the star has changed, the perfectly conducting crust carries a surface current where the Lorentz forces act. On the right, the crust is not perfectly conducting, having magnetic diffusivity $\eta$ and contains, after time $t$, a thin conducting layer (of thickness of order $\sqrt{t \eta}$ ).

crust freezes. To look at the stresses on the crust, therefore, we need to subtract from the Lorentz force that which was present at the moment of freezing.

If the field is of order $10^{15}$ Gauss, a typical length scale $\mathcal{L}$ is $1 \mathrm{~km}$ and the crust has a density of $10^{14} \mathrm{~g} \mathrm{~cm}^{-3}$ then the Lorentz force per unit mass will be around $B^{2} / 4 \pi \mathcal{L} \rho \sim 10^{8} \mathrm{~cm} \mathrm{~s}^{-2}$. The force of gravity per unit mass will be $G M / R^{2} \approx 2 \times 10^{14} \mathrm{~cm} \mathrm{~s}^{-2}$, which is balanced by pressure. The vertical component of the Lorentz force can therefore be balanced by the change in gravitational force brought about by a vertical displacement of just $0.3 \mathrm{~cm}$.

We feel it is safe to assume that the crust is flexible enough to accommodate vertical movements of this size without cracking. The vertical component of the Lorentz force, therefore, is not of any great interest to us. Of the remaining Lorentz force, that is, the horizontal component minus that at the moment of crust formation, it is only the part which can be expressed as the curl of a vector normal to the surface which interests us, as the other part can be supported by pressure forces. This is the part of the Lorentz force given by

$$
\begin{aligned}
& F_{x}=\frac{1}{4 \pi} \frac{\partial\left(B_{z} B_{x}\right)}{\partial z} \quad \text { and } \\
& F_{y}=\frac{1}{4 \pi} \frac{\partial\left(B_{z} B_{y}\right)}{\partial z} .
\end{aligned}
$$

This is then integrated over a zone from $r=0.79 R_{*}$ to $r=$ $0.93 R_{*}$ (the inner boundary of the crust is at $r=0.86 R_{*}$ ), and can be plotted as arrows on a 2-D projection of the star. The projection used is that where longitude forms the $x$-axis and latitude forms the $y$-axis; to do this an axis is of course required - we use the dipole axis of the radial component $B_{\mathrm{r}}$ of the field on the surface.

It is also useful to calculate an average Lorentz stress over the whole of the crust, to see how it changes with time. This is done by simply taking a root-mean-square of the component described above.

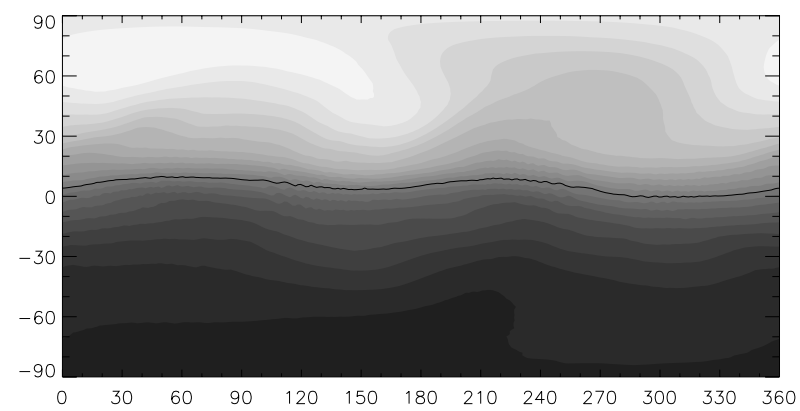

Fig. 7. Initial condition for the magnetar field evolution calculation, showing the radial component $B_{\mathrm{r}}$ of the magnetic field on the surface of the star, in longitude (horizontal) and latitude. Light (dark) shows positive (negative) $B_{\mathrm{r}}$. The black line shows the magnetic equator $B_{\mathrm{r}}=0$.

\subsection{Result}

The evolution of the field inside a star with a solid crust was modelled at a resolution of $96^{3}$, producing a crust 9 grid spacings thick. The code runs for about 20 Alfvén crossing times, corresponding to about $1 / 4$ of a diffusion time $R^{2} / \eta$, where $R$ is the radius of the star. Since the characteristic length scale of the torus configuration is about 3 times smaller (cf. Figs. 9 and 10), this corresponds to about 2 times the characteristic diffusion time of the torus configuration itself. After this time, the code crashes when the stresses at the surface become too large. This limitation does not matter to us, since we are only interested in looking at the buildup of stresses and not in the cracking process itself.

The initial conditions for the run are taken from the output from an earlier run in which an initial random field configuration evolved into a twisted torus (see Paper I). The radial component of the field on the surface is shown in Fig. 7. It can be seen that the field is chiefly dipole in nature. Figure 8 shows the modulus of the field, which is strongest just above the magnetic equator - the dipole field is slightly offset from the centre of the star.

Field line tracings are shown in Figs. 9 and 10. The twisted torus shape is clearly visible. 


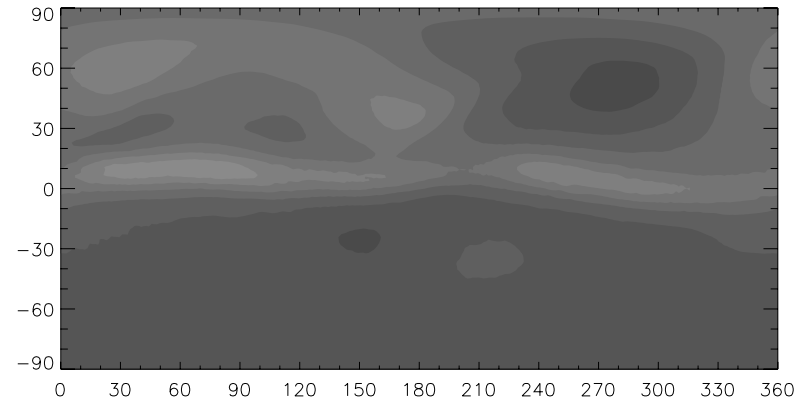

Fig. 8. As Fig. 7, showing the modulus of the magnetic field $|\boldsymbol{B}|$ on the surface of the star. Light (dark) represent regions of strong (weak) field.

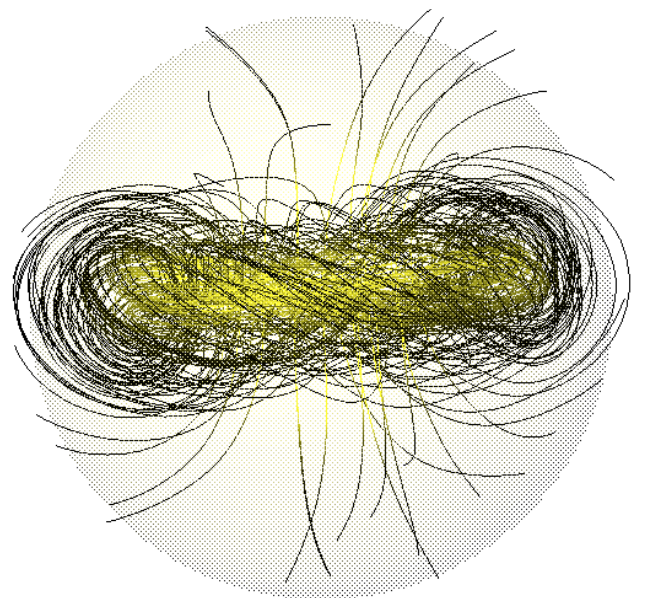

Fig. 9. Field lines at the beginning of the run, with the core of the torus configuration shown as a shaded surface. A transparent sphere representing the surface of the star is also plotted.

Figure 11 shows the rms stress per unit area as a function of time.

Figure 12 shows the horizontal components of the stress at the surface (as described in the previous section) at four times after the assumed formation of the crust at $t=0$, at roughly equal intervals. It can be seen that there are lines separating areas with their Lorentz stress in opposite directions (these show up as black lines between the bright areas on the right-handside of Fig. 12; they are mainly present in the equatorial zone). It is presumably along these lines that the fault lines will appear. There are also patterns where the Lorentz force is exerting a torque on a part of the the crust; this is visible mainly in the polar regions. Smaller length scales were still present in the initial configuration at a low amplitude; their more rapid diffusion causes the smaller scales seen in the stress pattern in Fig. 12, as well as in the somewhat faster initial development of the stress in Fig. 11.

\section{Application: scenario for magnetar evolution}

The results of this study lead logically to a model for magnetar evolution. First, we require the crust to have a much higher electrical conductivity than the interior, so the field is "frozen" into the crust while the field continues to evolve in the fluid interior. The magnetic diffusivity $\eta$ of the interior has to be such

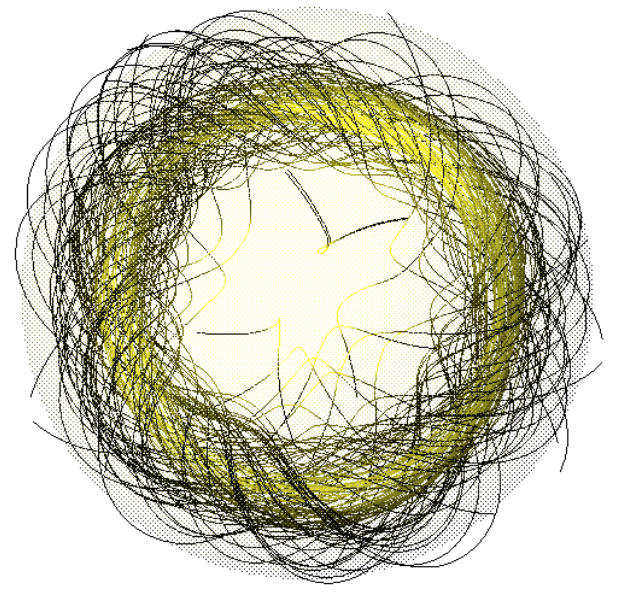

Fig. 10. As Fig. 9, but viewed from above.

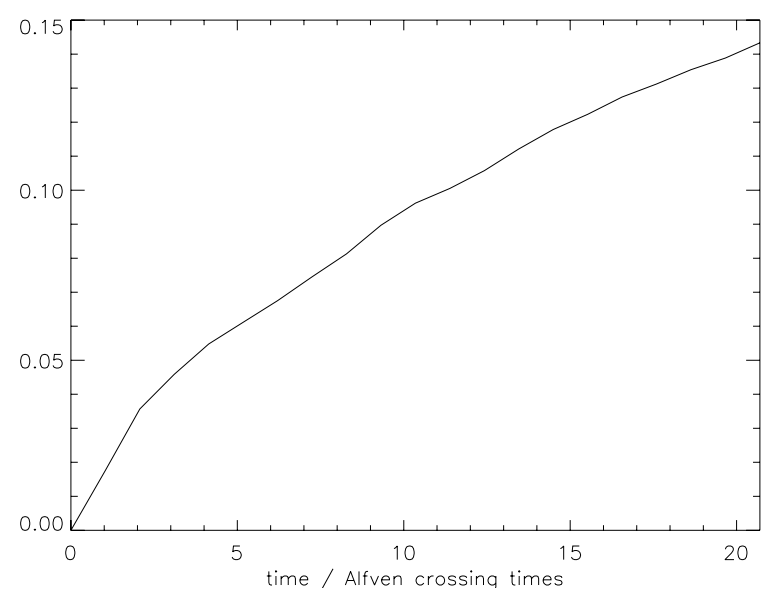

Fig. 11. Root-mean-square stress as a function of time. The unit used is the mean magnetic pressure $B^{2} / 8 \pi$ in the stellar interior at $t=0$.

that the timescale of the field's evolution, $\mathcal{L}^{2} / \eta$ (where $\mathcal{L}$ is a typical length scale), is of the order of $10^{4}$ years, the observed lifetime of these objects. The second requirement of the model is that the star contains a stable field at the moment when the crust starts to form, of the order of $10^{15}$ Gauss.

The hypothesis then is that this stable field in the fluid interior evolves under the influence of diffusion, while the field in the crust is held relatively constant by higher conductivity. This causes stress to build up in the lower part of the crust, which at some point becomes strong enough to cause fractures to appear, and energy is released by two mechanisms. In the movement of the crust, magnetic energy is converted to kinetic energy and then to heat. In the atmosphere, the movement of different parts of the crust causes the field lines to become twisted, giving rise to large currents in the tenuous medium. This results in Ohmic heating and eventual relaxation back to a potential field.

\section{Summary}

We have studied the release of magnetic energy in a strongly magnetised neutron star, with a view to finding an explanation for the high luminosity of the soft gamma repeaters and anomalous X-ray pulsars. These objects are observed to release energy over a timescale of around $10^{4}$ years, much greater 

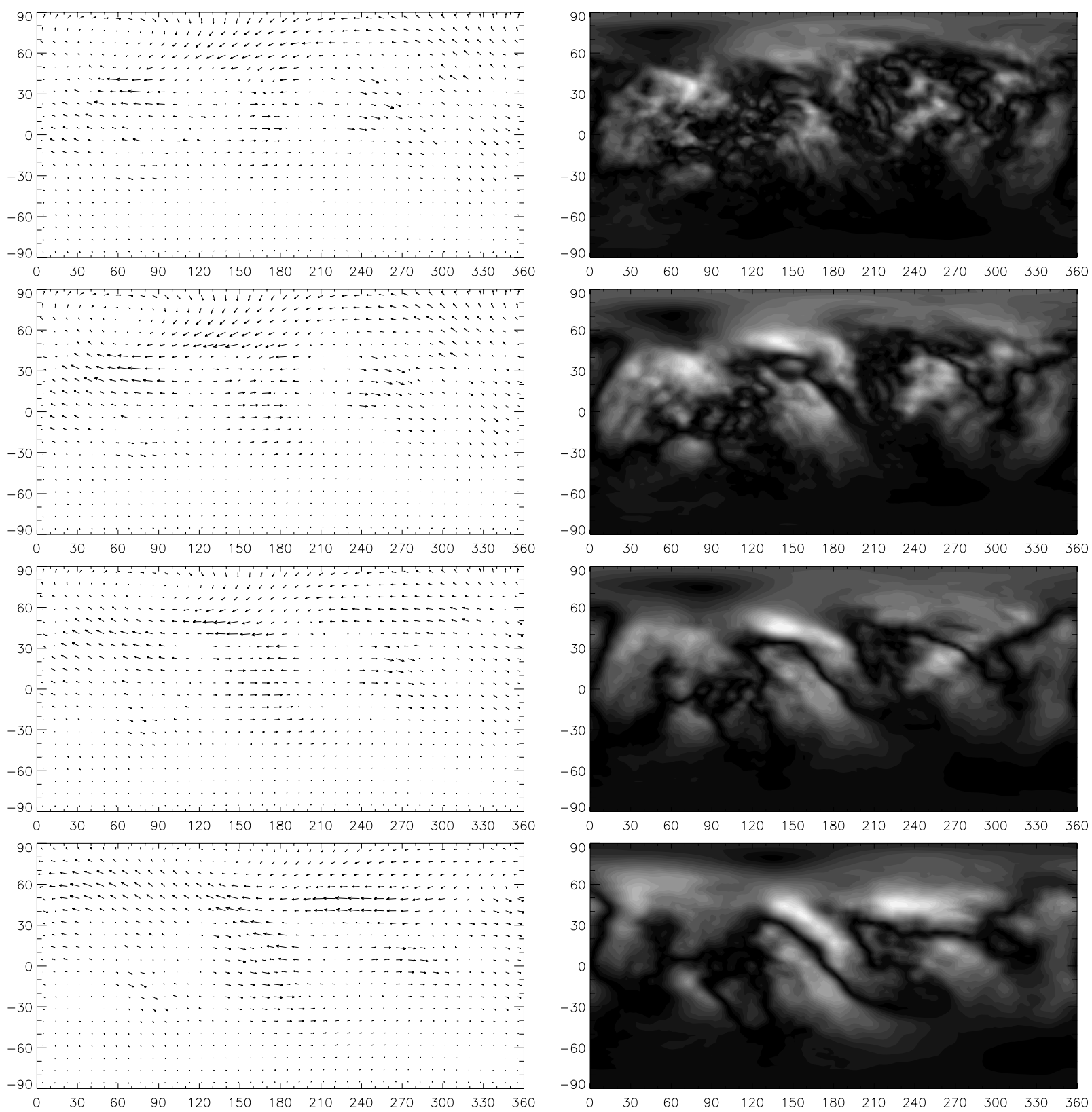

Fig. 12. Maps of stress at the outer surface, at four roughly equal temporal intervals: $t=5.3,10.4,15.6,20.7$ Alfvén crossing-times respectively from top to bottom. On the left hand side, arrows show the direction and strength of the Lorentz stress. The right hand side shows the magnitude of the Lorentz stress. (Arrows and grey scales are scaled to maximum over the surface in each panel.)

than the Alfvén timescale (the time taken for an Alfvén wave to travel across the star, $0.1 \mathrm{~s}$ ) on which an unstable field evolves. This leads us to the conclusion that the magnetic field must go through a sequence of quasi-static, stable equilibria, possibly occasionally punctuated by instability. A central question is thus what this stable magnetic equilibrium looks like and how it can form.

By following the decay of arbitrary initial field configurations with 3D magnetohydrodynamic simulations we have shown in Paper I that a stable configuration exists in the form of a torus of linked poloidal-toroidal field, confirming educated guesses existing in the literature (e.g. Prendergast 1956). We found there that in all cases, the field either reaches this torus field, or it decays to nothing; no other end-states were found. Whether the field evolves into the stable torus shape was found to depend on how concentrated the field is in the centre of the star. A field concentrated in the stellar core can reach the torus configuration, whilst a field whose energy distribution extends to greater radii and into the atmosphere usually decays to nothing.

This result can be understood in terms of helicity conservation. In the absence of reconnection, helicity is a conserved quantity, so that an arbitrary field will evolve to the lowest energy state with the same helicity. This appears to be the linked poloidal-toroidal torus. If the initial field is buried less deeply in the star and has too strong a contact with the atmosphere, 
an environment where reconnection cannot be ignored, helicity conservation is no longer valid and both the helicity and field energy can decay to zero.

A configuration considered before in connection with the stability of magnetic fields in neutron stars is a uniform field inside matching to a pure dipole outside the star. The instability argument of Flowers \& Ruderman (1977) shows that the energy of this configuration is reduced by rotating one half of the star by $180^{\circ}$ with respect to the other along a magnetic surface. Our numerical simulation of this configuration confirms its instability, but instead of a large scale rotation, the instability is found to take place at higher azimuthal wavenumbers, with displacements reminiscent of other interchange instabilities such as Rayleigh-Taylor.

The Flowers-Ruderman field has zero helicity, hence, by a symmetry argument we may expect that it will not develop the stable tori found in Paper I. A torus must have an axis, which can only be parallel to the original axis; the toroidal component must then go around this axis in one direction or the other, but there is no way to decide which direction. However, this argument does not entirely rule out the existence of stable fields with zero helicity as end states of unstable evolution. For instance two tori on top of each other, with opposite "handedness". It is also possible that the interior could acquire some helicity by loss of helicity through the surface of the star in the course of the evolution of the field. We have seen no evidence of this occurring, however, and our tentative conclusion is that the decay of a Flowers-Ruderman configuration may well be rather complete.

We have studied the slow evolution of a stable torus configuration in the diffusive interior of a neutron star, underneath a solid and perfectly-conducting crust. As the field changes under the influence of diffusion, stress develops in the crust. We find that this stress is dominated by patterns that would cause strong rotational displacements on the neutron star surface when released, consistent with the model of SGR outbursts developed by Thompson \& Duncan (1995, 2001).

One may speculate what the final evolution of a magnetar field would be as this process continues. In Paper I we found that the diffusing torus gradually loses the azimuthal field component that provides stability to the torus, by loss through the surface of the star. At some point, this configuration, dominated by the poloidal component, became unstable in the way predicted by the Flowers-Ruderman argument, developing an $m=2$ distortion. The further evolution of this configuration led to a final episode of rapid decay. If this also happens in magnetars, it predicts a final episode of rapid decay, perhaps in the form of a giant outburst, as suggested before by Eichler (2002).
Acknowledgements. We thank the referee for his suggestions and valuable criticism of an earlier version of the text.

\section{References}

Acheson, D. J. 1978, Phil. Trans. Roy. Soc. Lond., A289, 459

Blandford, R. D., Applegate, J. H., \& Hernquist, L. 1983, MNRAS, 204, 1025

Braithwaite, J. 2005, to be published [arXiv: astro-ph/0512182]

Braithwaite, J., \& Nordlund, Å. 2006, A\&A, 450, 1077 (Paper I)

Braithwaite, J., \& Spruit, H. C. 2004, Nature, 431, 891

Cline, T. L. 1982, Gamma ray transients and related astrophysical phenomena (AIP), ed. Lingenfelter et al., 17

Duncan, R. C., \& Thompson, C. 1992, ApJ, 392, L9

Eichler, D. 2002, MNRAS, 335, 883

Flowers, E., \& Ruderman, M. A. 1977, ApJ, 215, 302

Hurley, K., Kouveliotou, C., Woods, P., et al. 1999, ApJ, 510, L107

Kouveliotou, C., Dieters, S., Strohmayer, T., et al. 1998, Nature, 393, 235

Low, B. C. 2001, JGR, 106, 25141

Markey, P., \& Tayler, R. J. 1973, MNRAS, 163, 77

Markey, P., \& Tayler, R. J. 1974, MNRAS, 168, 505

Mazets, E. P., et al. 1999, Astron. Lett., 25, 635

Mereghetti, S. 2000, in The neutron star - black hole connection, ed. Kouveliotou, Paradijs, \& Ventura, 351

Moffat, K. H. 1990, private communication

Nordlund, Å., \& Galsgaard, K. 1995, http://www . astro.ku.dk/ aake/papers/95.ps.gz

Palmer, D. M., Barthelmy, S., Gehrels, N., et al. 2005, Nature, 434, 1107

Pitts, E., \& Tayler, R. J. 1986, MNRAS, 216, 139

Prendergast, K. H. 1956, ApJ, 123, 498

Rothschild, R. E., Kulkarni, S. R., \& Lingenfelter, R. E. 1994, Nature, 368,432

Shapiro, S. L., \& Teukolsky, S. A. 1983, Black holes, white dwarfs and neutrons stars: the physics of compact objects (John Wiley \& Sons)

Spruit, H. C. 2002, A\&A, 381, 923

Tayler, R. J. 1973, MNRAS, 161, 365

Thompson, C., \& Duncan, R. C. 1993, ApJ, 408, 194

Thompson, C., \& Duncan, R. C. 1995, MNRAS, 275, 255

Thompson, C., \& Duncan, R. C. 1996, ApJ, 473, 322

Thompson, C., \& Duncan, R. C. 2001, ApJ, 561, 980

Woltjer, L. 1958, Proc. Nat. Acad. Sci., 44, 489

Woods, P. M., Kouveliotou, C., Finger, M. H., et al. 2000, ApJ, 535, L55

Wright, G. A. E. 1973, MNRAS, 162, 339

Zhang, M., \& Low, B. C. 2003, ApJ, 584, 479 